\title{
Hanbury Brown and Twiss Correlations of Cooper Pairs in Helical Liquids
}

\author{
Mahn-Soo Choi* \\ Department of Physics, Korea University, Seoul 136-713, Korea \\ School of Physics, Korea Institute for Advanced Study, Seoul 130-722, Korea and \\ Asia Pacific Center for Theoretical Physics, Pohang 790-784, Korea
}

(Dated: April 26, 2022)

\begin{abstract}
We propose a Hanbury Brown and Twiss (HBT) experiment of Cooper pairs on the edge channels of quantum spin Hall insulators. The helical edge channels provide a well defined beam of Cooper pairs and perfect Andreev reflections from superconductors. This allows our setup to be identical in spirit to the original HBT experiment. Interestingly, the cross correlation is always negative and provides no hint of the bosonic nature of Cooper pairs. This counter-intuitive result is attributed to the perfect Andreev reflection and the true beam splitter in the septup.

PACS numbers: 73.23.Ad; 73.63.-b; 03.75.Hh
\end{abstract}

\section{INTRODUCTION}

The Bose-Einstein condensation of constituent particles results in superfluidity in bosonic systems.[1] Superconductivity in electronic systems is attributed to the pairing of electrons into so called "Cooper pairs".[2] It is thus plausible to expect Cooper pairs to bear some bosonic nature, and to regard superconductivity as a condensation. Nevertheless, mathematically, Cooper pairs are not pure bosons because the pair creation and annihilation operators do not obey strictly the boson commutation relations, [2] and the bosonic nature of Cooper pairs still remains controversial. Therefore, it will be invaluable to examine the issue directly in experiments. Here we propose a Hanbury Brown and Twiss (HBT) experiment of Cooper pairs on the edge channels of quantum spin Hall insulators, $[3,4]$ a recently discovered new state of matter. Surprisingly,[5] the cross correlation is always negative and shows no hint of the bosonic nature of Cooper pairs once they are emitted from the superconductor. Previously, theoretical[6$9]$ and experimental[10, 11] works showed positive or negative correlation depending on the system parameters. Interestingly, the cross correlation has been predicted to be always negative in a diffusive multi-terminal superconductor-normal-metal contacts.[12]

A HBT effect is an intensity interference between two partial beams. It was originally introduced in order to overcome the technical difficulties in measuring the size of stars with Michelson interferometers. After the pioneering experiment by HBT in 1956,[13, 14] it was soon realized that the effect can determine quantum-statistical properties of a stream of particles:[15, 16] The intensity correlation is positive for bosons (obeying Bose-Einstein statistics) while negative for fermions (obeying FermiDirac statistics).[17]

An ideal HBT experiment requires a well-defined beam of particles and a tunable beam splitter (BS). For normal electrons, an electron beam is achieved on the chiral edge states of an integer quantum Hall insulator (IQHI), for which a quantum point contact (QPC) serves as a beam splitter.[18, 19] It does not work for Cooper pairs because the high magnetic field required for IQHI breaks them.

Quantum spin Hall insulator (QSHI) is a prototype example of topological insulators. Topological insulators are characterized by bulk excitation energy gap and gapless edge modes, the latter being intimately connected to the topological nature.[3, 20] In QSHIs, the spin-orbit coupling gives the edge modes a helical feature, spin up $(\uparrow)$ electrons moving to the right and spin down $(\downarrow)$ electrons moving to the left, and the time-reversal invariance prevents back scattering even in the presence of disorder. [3, 20] The helical edge states are thus a Kramers pair and duplicate copies of a chiral edge mode. Then it is clear that a QPC serves as a BS for helical edge modes.[21, 22]

When a superconductor are put on top of helical edge modes, the edge modes become superconducting due to the proximity effect. [23, 24] The edge modes beneath the superconductor will be referred to superconducting edge modes or simply "superconductors". A Cooper pair, itself being a pairing between time-reversal counterparts, moves with no back scattering across the (point) interface between superconductor and helical edge modes. That is, the Andreev reflection is always perfect even in the presence of interface barrier.[25] This is in sharp contrast to usual normal-superconductor hybrid structures, where in reality normal electron reflection is unavoidable. Therefore, the helical edge states provide a well-defined and transparent channel for Cooper pairs.[26] Two-dimensional QSHI has been observed recently in $\mathrm{HgTe} / \mathrm{CdTe}$ quantum wells[4] and also expected in InAs/GaSb/AlSb type-II quantum wells.[27]

Several HBT-type experiments of Cooper pairs have been proposed before.[6-9] In their cases, however, a $Y$-junction is used instead of a true electron BS, and not only Andreev but also normal-electron reflections are involved.[8] It is shown below that these differences affect significantly the results. Our setup is much closer in spirit to the original HBT experiment. Another closely 
(a)

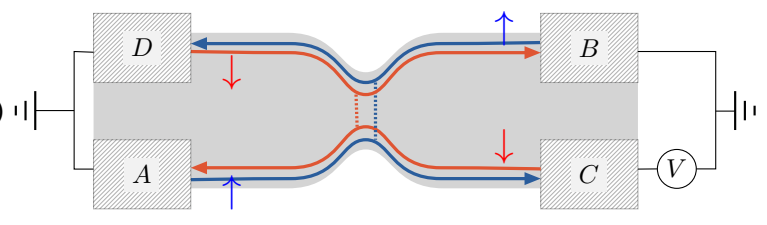

(b)

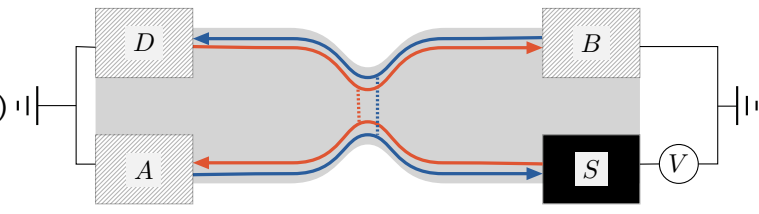

(c) 1

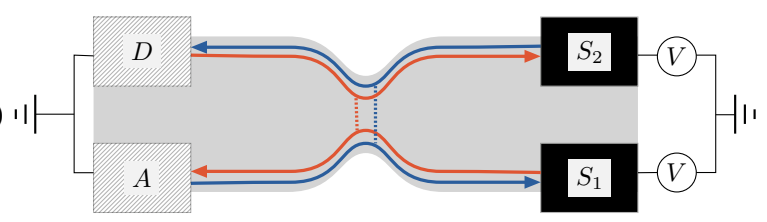

FIG. 1. The schematic setups for HBT experiment of Cooper pairs using edge states of QSHI. The light gray represent the QSHI sample, the arrowed thick lines represent the helical edge states. $\uparrow(\downarrow)$ electrons move counterclockwise (clockwise). The QPC formed by constriction with side gates serves as an electron beam splitter. A HBT experiment setup (a) for normal electrons and (b) for Cooper pairs. In (c), Cooper pairs are injected from two superconductors.

related work is Ref. 28: Here the Cooper pairs were injected to helical edge modes of QSHI through direct tunneling from spin-singlet superconductors. Unlike in our setup, the coupling between the helical edge modes and the superconductor in their setup is weak. As pointed out by the authors, [28] most electrons are thus passing along the edges without Andreev scattering and rare electrons do undergo Andreev reflection but scatter to a different edge as holes. Such a crossed Andreev reflection leads to almost perfect positive correlation.[28]

Recently, the splitting of Cooper pairs has been demonstrated experimentally.[10, 11] However, in these experiments the scattering of Cooper pairs themselves have been suppressed with Coulomb interaction. While the experiments are only conductance measurement as they are, the cross correlation is therefore expected manifestly positive in such setups.[29]

\section{NORMAL-ELECTRON CASE}

We consider a stripe of QSHI. The helical edge states at its boundary are described by a one-dimensional Dirac Hamiltonian

$$
H=\hbar v \sum_{\ell=1,2} \int d x\left[\psi_{\ell \downarrow}^{\dagger}\left(i \partial_{x}-\mu\right) \psi_{\ell \downarrow}-\psi_{\ell \uparrow}^{\dagger}\left(i \partial_{x}+\mu\right) \psi_{\ell \uparrow}\right]
$$

where $\psi_{\uparrow}\left(\psi_{\downarrow}\right)$ is the field operator of $\uparrow(\downarrow)$ electrons, $\mu$ is the chemical potential, and $v$ is the propagation velocity. The edge of a QSHI sample forms a closed loop

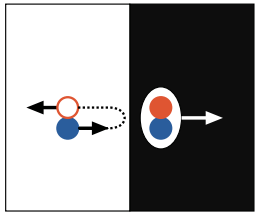

(a)

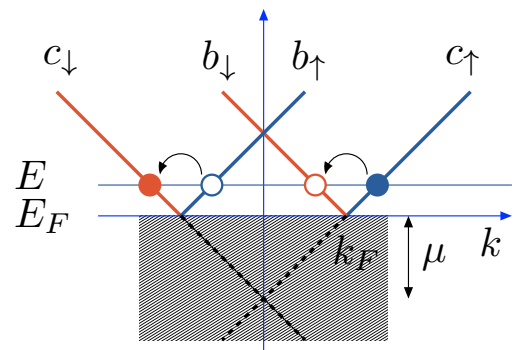

(b)
FIG. 2. (a) Andreev reflection at the interface between normal (left) and superconducting (right) helical edge modes, where $\uparrow$ electron is reflected as a $\downarrow$ hole. (b) The energy-momentum relation of particles $\left(c_{\sigma}\right)$ and holes $\left(b_{\sigma}\right)$ with spin $\sigma=\uparrow, \downarrow$.

and can never be terminated in the middle. In Eq. (1) it has been assumed that the lower $(\ell=1)$ and upper $(\ell=2)$ segment of the whole edge are isolated from the rest by putting contact reservoirs $A, B, C, D$, and other additional contacts (not shown) as in Fig. 1 (a).

Constricting the QSHI bar with side gates forms a QPC. The $\uparrow$ electron injected to the QPC from reservoir $A$, for example, either moves on to reservoir $C$ with probability amplitude $t$ or tunnels to the upper edge going out to reservoir $D$ with probability amplitude $r$. The QPC thus serves as a tunable BS for electrons. The QPC is thus characterized completely by the scattering matrix $\Sigma$

$$
\left[\begin{array}{c}
c_{1 \downarrow}^{\prime} \\
c_{2 \downarrow}^{\prime} \\
c_{1 \uparrow}^{\prime} \\
c_{2 \uparrow}^{\prime}
\end{array}\right]=\Sigma\left[\begin{array}{c}
c_{1 \uparrow} \\
c_{2 \uparrow} \\
c_{1 \downarrow} \\
c_{2 \downarrow}
\end{array}\right]=\left[\begin{array}{llll}
0 & 0 & t & r \\
0 & 0 & r & t \\
t & r & 0 & 0 \\
r & t & 0 & 0
\end{array}\right]\left[\begin{array}{c}
c_{1 \uparrow} \\
c_{2 \uparrow} \\
c_{1 \downarrow} \\
c_{2 \downarrow}
\end{array}\right]
$$

relating the amplitudes $c_{\ell \sigma}^{\prime}(\ell=1,2$ and $\sigma=\uparrow, \downarrow)$ at the output ports to $c_{\ell \sigma}$ at the input ports of QPC. We have suppressed the energy (or momentum) dependence of $c_{\ell \sigma}$ for notational simplicity.

We apply a bias voltage $V$ to reservoir $C$, keeping $A$, $B$, and $D$ electrically grounded. With this bias configuration, only $\downarrow$ electrons are injected from $C$ along the lower edge. The quantum statistical properties of the electron beam are characterized by the correlation functions defined by

$$
S_{\alpha \beta}=\int_{-\infty}^{\infty} d t\left\langle\Delta I_{\alpha}(t) \Delta I_{\beta}(0)+\Delta I_{\beta}(0) \Delta I_{\alpha}(t)\right\rangle
$$

with $\Delta I_{\alpha}(t)=I_{\alpha}(t)-\bar{I}_{\alpha}$, where $\bar{I}_{\alpha}$ is the average current into reservoir $\alpha$. Given the scattering matrix in (2), the calculation of $\bar{I}_{\alpha}$ and $S_{\alpha \beta}$ is a simple application of the Landauer-Büttiker formalism.[18] We focus on the zerotemperature limit, $k_{B} T \ll e V$. In the present case, the average currents are given by

$$
\bar{I}_{A}=\frac{e^{2} V}{2 \pi \hbar}|t|^{2}, \quad \bar{I}_{B}=\frac{e^{2} V}{2 \pi \hbar}|r|^{2} .
$$


Clearly the injected current $\bar{I} \equiv-\bar{I}_{C}$ flows either to $A$ or $B\left(I_{A}+I_{B}=\bar{I}\right)$, but not to $D\left(\bar{I}_{D}=0\right)$. This partitioning at the BS leads to the well-known results[18]

$$
S_{A A}=S_{B B}=-S_{A B}=2 e \bar{I}|r t|^{2}
$$

for the current correlations, and its negative value is well understood by their antibunching behavior.[18] For later reference, we stress that the contact $D$ is completely "mute", i.e., $I_{D}=0$ and $S_{A D}=S_{B D}=S_{D D}=0$.

\section{HBT CORRELATIONS OF COOPER PAIRS}

Let us now turn to the setup, Fig. 1 (b), of our main concern. We replace the normal contact $C$ by a superconducting contact $S$. A superconducting edge state is achieved by putting an $s$-wave superconductor on top of the edge. Due to the proximity effect, the edge states beneath the superconductor form a superconducting state with an induced gap $\Delta=\Delta_{0} e^{-i \varphi}$.[24] The Hamiltonian of the superconducting edge modes is then given by [cf. (1)]

$$
\begin{array}{r}
H_{S}=\hbar v \int d x\left[\psi_{1 \downarrow}^{\dagger}\left(i \partial_{x}-\mu\right) \psi_{1 \downarrow}-\psi_{1 \uparrow}^{\dagger}\left(i \partial_{x}+\mu\right) \psi_{1 \uparrow}\right. \\
\left.+\Delta \psi_{1 \uparrow}^{\dagger} \psi_{1 \downarrow}^{\dagger}+\Delta^{*} \psi_{1 \downarrow} \psi_{1 \uparrow}\right]
\end{array}
$$

We apply bias voltage $V$ to $S$ and keep $A, B$, and $D$ grounded, effectively injecting Cooper pairs from $S$ along the lower edge channels. The injection of Cooper pairs can be equivalently described by the Andreev reflection process, where say an $\uparrow$ electron injected towards superconductor is reflected by a $\downarrow$ hole away from superconductor, see Fig. 2. The Andreev reflection amplitude is given by $a=e^{i \varphi-i \arccos \left(E / \Delta_{0}\right)}$ for incident electrons of energy $E\left(|E|<\Delta_{0}\right)$. Note that due to the time-reversal invariance the Andreev reflection probability remains unity, $|a|^{2}=1$, regardless of the imperfections at the edgesuperconductor interface.[25] This is in stark contrast to the usual normal-superconductor interface, where interface imperfections suppress Andreev reflections.

Combining the Andreev reflections at the normalsuperconductor interface and the normal scattering (2) at the QPC, one obtains the total scattering matrix for electrons and holes:

$$
\left[\begin{array}{c}
c_{1 \downarrow}^{\prime} \\
b_{1 \downarrow}^{\prime} \\
c_{2 \downarrow}^{\prime} \\
b_{2 \downarrow}^{\prime} \\
c_{2 \uparrow}^{\prime} \\
b_{2 \uparrow}^{\prime}
\end{array}\right]=\left[\begin{array}{cccccc}
0 & a^{*}|t|^{2} & 0 & a^{*} r^{*} t & r & 0 \\
a|t|^{2} & 0 & a r t^{*} & 0 & 0 & r^{*} \\
0 & a^{*} r t^{*} & 0 & a^{*}|r|^{2} & t & 0 \\
a r^{*} t & 0 & a|r|^{2} & 0 & 0 & t^{*} \\
r & 0 & t & 0 & 0 & 0 \\
0 & r^{*} & 0 & t^{*} & 0 & 0
\end{array}\right]\left[\begin{array}{c}
c_{1 \uparrow} \\
b_{1 \uparrow} \\
c_{2 \uparrow} \\
b_{2 \uparrow} \\
c_{2 \downarrow} \\
b_{2 \downarrow}
\end{array}\right]
$$

where $b_{\ell \sigma}$ and $b_{\ell \sigma}^{\prime}(\ell=1,2$ and $\sigma=\uparrow, \downarrow)$ are amplitudes of the holes on the input and output ports. Here $r^{*} \equiv$
$[r(-E)]^{*}, t^{*} \equiv[t(-E)]^{*}$, and $a^{*} \equiv[a(-E)]^{*}$ describe the scattering of holes. We ignore weak energy dependence of $r$ and $t$.

The average currents $I_{\alpha}$ and the current correlations $S_{\alpha \beta}(\alpha, \beta=A, B, D)$ are calculated using the Landauer-Buttiker formalism extended to the normalsuperconductor hybrid structure.[30, 31] The currents

$$
\bar{I}_{A}=\frac{2 e^{2} V}{2 \pi \hbar}|t|^{2}, \quad \bar{I}_{B}=\frac{2 e^{2} V}{2 \pi \hbar}|r|^{2},
$$

are twice larger than (4), demonstrating perfect Andreev reflections at (or injection of Cooper pairs from) $S$. The correlation functions are also given exactly in the same form as (5),

$$
S_{A A}=S_{B B}=-S_{A B}=2 e \bar{I}|r t|^{2},
$$

except that the total current $\bar{I} \equiv \bar{I}_{A}+\bar{I}_{B}$ is now twice larger. Surprisingly, the cross correlation is negative. (As in the normal case above, the contact $D$ is mute; $I_{D}=0$ and $\left.S_{A D}=S_{B D}=S_{D D}=0\right)$.

Why is it surprising? The description in (7) in terms of electrons and holes is equivalent to Cooper pairs injected from $S$ and scattered at QPC. Note that either (i) entire Cooper pairs go to $A$ or $B$ [Fig. 3 (a) and (c)], or (ii) constituent electrons in each pair split up into $A$ and $B$ [Fig. 3 (b) and (d)]. Naively, one may expect a positive contribution from case (ii) with one electron at each port $A$ and $B$ simultaneously. Assuming (partial) bosonic nature of Cooper pairs, one may also expect $S_{A D}>0$ in case (i).

The above naive expectation fails because it has ignored the two-particle interference.[32] For example, the two process ( $A$ 个-electron; $B$ 个-electron $) \rightarrow(A \downarrow$-hole; $B \downarrow$-hole) [Fig. 3 (a) and (c)] and ( $A$ 个-electron; $B \uparrow$ electron $) \rightarrow(B \downarrow$-hole; $A \downarrow$-hole $)[$ Fig. 3 (b) and (d)] are not distinguishable and interfere with each other. Due to the Fermi-Dirac statistics, the amplitudes for these processes are opposite in sign, hence giving negative cross correlation in (9).

There is another simple way to understand the negative correlation. For example, $\uparrow$ electron from $A$ undergoes either Fig. 3 (a) or (b). Since the perfect Andreev reflection at the edge-superconductor interface is noiseless, it is nothing but the partitioning of a single hole and thus gives a negative contribution to $S_{A B}$. Similarly, the partitioning into Fig. 3 (c) and (d) also give negative contributions.

We thus conclude that Cooper pairs bear no bosonic characters at all, once they get out of the superconductor. It is interesting to note that in recent experiments with quantum dots[10, 11] the processes Fig. 3 (a) and (c) have been suppressed due to Coulomb interactions. In this case, there is no two-particle interference. 


\section{BACK-SCATTERING EFFECT}

The above conclusion appears contradictory to the previous theoretical works, [6-9] where positive correlation was predicted in certain range. As mentioned earlier, in these works a $Y$ junction is used instead of a true electron BS. One crucial effect is the multiple reflections between the junction and the superconductor. Further, not only Andreev but also normal-electron reflections are involved. The latter effect cannot be simulated in our system and is analyzed in the supplementary material.

To simulate the former effects, let us now replace both $C$ and $B$ by superconductors, $S_{1}$ and $S_{2}$, with phases $\varphi_{1}$ and $\varphi_{2}$, respectively. We apply bias voltage $V$ on both $S_{1}$ and $S_{2}$ and keep $A$ and $D$ grounded. The scattering of electrons and holes is governed by the scattering matrix (ignoring corrections of order $E^{2} / \Delta_{0}^{2} \ll 1$ )

$$
\left[\begin{array}{c}
c_{1 \downarrow}^{\prime} \\
b_{1 \downarrow}^{\prime} \\
c_{2 \uparrow}^{\prime} \\
b_{2 \uparrow}^{\prime}
\end{array}\right]=\left[\begin{array}{cccc}
0 & \frac{a_{1}^{*}|t|^{2}}{z^{*}} & \frac{w^{*} r}{z^{*}} & 0 \\
\frac{a_{1}|t|^{2}}{z} & 0 & 0 & \frac{w r^{*}}{z} \\
\frac{w r}{z} & 0 & 0 & \frac{a_{2}^{*}|t|^{2}}{z} \\
0 & \frac{w^{*} r^{*}}{z^{*}} & \frac{a_{2}|t|^{2}}{z^{*}} & 0
\end{array}\right]\left[\begin{array}{c}
c_{1 \uparrow} \\
b_{1 \uparrow} \\
c_{2 \downarrow} \\
b_{2 \downarrow}
\end{array}\right]
$$

where $a_{1}=-e^{i \varphi_{1}+i 2 k_{F} L_{1}}, a_{2}=-e^{i \varphi_{2}-i 2 k_{F} L_{2}}, k_{F}=$ $\mu / \hbar v$, and $L_{1}$ and $L_{2}$ are the distances from the QPC to the superconducting contacts $S_{1}$ and $S_{2}$, respectively. The multiple reflections between QPC and superconductors are manifested through the factors $z=1-|r|^{2} e^{i \theta}$ and $w=1-e^{i \theta}$, where $\theta=\left(\varphi_{1}-\varphi_{2}\right)+2 k_{F}\left(L_{1}+L_{2}\right)$ is the phase accumulation during one cycle of the multiple reflections. This immediately leads to the resonance behavior of the average currents,

$$
\bar{I}_{A}=\bar{I}_{D}=\frac{2 e^{2} V}{2 \pi \hbar} \frac{|t|^{4}}{1+|r|^{4}-2|r|^{2} \cos \theta}
$$

and the correlations

$$
S_{A A}=S_{D D}=S_{A D}=2 e \bar{I} \frac{|r|^{2}(1-\cos \theta)}{1+|r|^{4}-2|r|^{2} \cos \theta}
$$

For $\theta=2 \pi n$ with $n$ an integer, the currents take maximum and the noises vanish.

We note that the sign of the cross correlation $S_{A D}$ is now positive, in contrast to (9), which is always negative. This difference is ascribed to the multiple reflections between QPC and superconductors. As noted by Baym,[33] the intensity correlation $\langle I(t+\tau) I(t)\rangle$ is equivalent to the relative probability to observe two particles at two points separated by a distance $v \tau$, where $v$ is the flight velocity. If the HBT experiment is done with a "true BS" as in Fig. 1 (b), then the intensity correlation is entirely due to the spatial distribution and the quantum statistical property of the particles in the beam itself. To the contrary, if the BS is replaced by a $Y$-junction[69] and multiple reflections occur between the source and

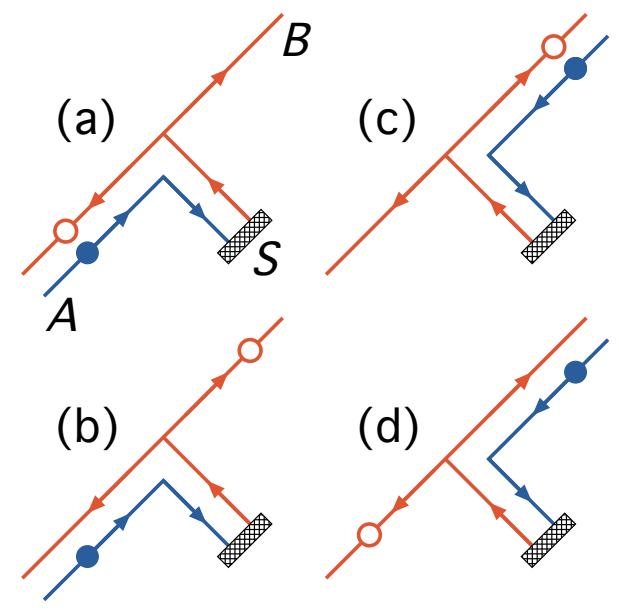

FIG. 3. Elementary scattering processes in a Hanbury Brown and Twiss experiment of Cooper pairs, described in electronhole picture. Blue (red) line represents the edge channels of $\uparrow(\downarrow)$ electrons (filled circle) or holes (empty circle). In Cooper pair picture, panel (a) corresponds to Cooper pairs being injected from $S$, scattered at the quantum point contact (QPC), and going entirely to $A$. Analogously, in (c), Cooper pairs entirely go to $B$. In (c) and (d), constituent electrons in a Cooper pair split up at the QPC.

the junction, then the cross correlation is not directly related to the spatial distribution in the beam alone but affected significantly by the successive interactions with the source. Therefore, the positive cross correlations in Refs. [6-9] do not represent entirely the quantum statistical properties of Cooper pairs after emitted from superconductor (away by distance larger than the superconducting coherence length).

This work was supported by the NRF grant (20110012494) and the BK21 program. The author expresses thanks to R. Aguado, A. Levy Yeyati, and R. Zambrini for useful discussions, and special thanks to M. Büttiker for helful comments and clarifying a few issues.

\section{Comparison of the Chaotic Quantum Dot and the Helical Liquid}

Previous theoretical works $[8,34]$ considered a chaotic quantum dot coupled to two normal leads 2 and 1 (corresponding to $A$ and $B$ in our case) and one superconducting reservoir. They found both positive and negative cross correlations in a wide range of parameter values, and provide clear interpretations of the relevant microscopic processes. Therefore, it will be useful for a deeper understanding of the physics behind the cross correlation to compare more closely the processes in their and our setup.

The crucial differences are (i) the multiple reflections between the dot-superconductor interface and the $Y$-junction (dot-normal-metal interface), and (ii) the 
normal-electron reflections (other than Andreev reflections). With the former effect discussed in the main text of the paper, here we focus on the latter effect.

Here we follow the scattering-matrix approach in [8], which provides a clear picture of the two-particle interference [32] and hence direct comparison of their system to ours. A semiclassical analysis is given in [34], which is more useful for interpretation in terms of partitioning noise.

We first discuss the case with the transparency $\Gamma_{S}$ of the dot-superconductor is perfect $\left(\Gamma_{S}=1\right)$. The normal and superconducting contacts supports $N$ and $M$ channels, respectively.

For small $2 N / M \ll 1$, the dominant process is the (local) Andreev reflections at the normal contact-dot interface, due to the gap in the dot spectrum induced by the superconductor. For finite (but small) $2 N / M$, there are finite probabilities of normal reflections. The cross Andreev reflections (CAR) from one normal reservoir to the other is still negligible; i.e., the terms such as $\left(S_{12}^{e h}\right)$ can be ignored. In this limit, the cross correlation is given by

$\frac{P_{12}}{4 e^{3} V / 2 \pi \hbar} \approx \frac{P_{12}^{e h}+P_{12}^{h e}}{4 e^{3} V / 2 \pi \hbar} \approx 2 \operatorname{Tr}\left[\left(S_{12}^{e e}\right)^{\dagger}\left(S_{11}^{e h}\right)\left(S_{21}^{h h}\right)^{\dagger}\left(S_{22}^{h e}\right)\right]$

This term arises from the two-particle interference of the two processes (a) and (b) in Fig. 4: The two processes cannot be distinguished and therefore the amplitudes (not probability) should be summed. The interference gives positive cross correlation [32] since it involves the exchange of electron and hole, namely, different species of particles. Therefore, the normal scattering processes (either electron or hole) in Fig. 4 is crucial to the positive cross correlation in this regime. In our case, the process of Fig. 4 is prohibited because of the helical (chiral) nature of the edge states.
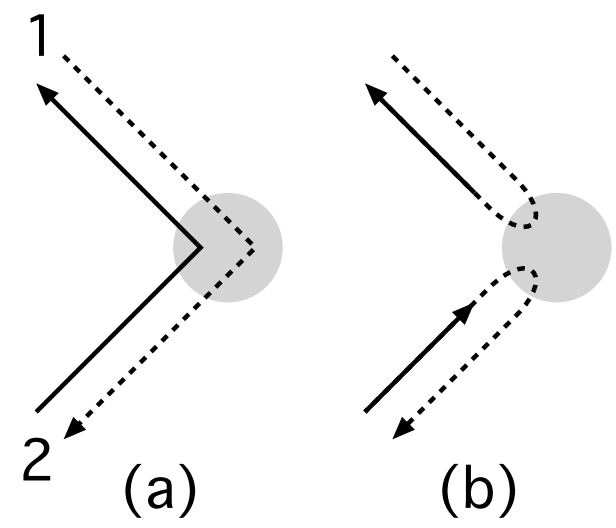

FIG. 4. Equation (13) corresponds to the interference of the two processes (a) and (b). The interference is positive since it only involves the exchange of electron and hole, i.e., different species of particles.

In the limit of $2 N / M \gg 1$, [8] obtained negative cross correlation. In this limit, there are finite probabilities of the CAR processes. According to [8], the main contributions come from $P_{12}^{e e}$ and $P_{12}^{h h}$, where $P_{12}^{e e}$ is expressed explicitly as

$$
\begin{aligned}
-\frac{P_{12}^{e e}}{4 e^{3} V / 2 \pi \hbar}=\operatorname{Tr}\left[\left(S_{11}^{e e}\right)^{\dagger}\left(S_{11}^{e h}\right)\left(S_{21}^{e h}\right)^{\dagger}\left(S_{21}^{e e}\right)+\left(S_{11}^{e e}\right)^{\dagger}\left(S_{12}^{e h}\right)\left(S_{22}^{e h}\right)^{\dagger}\left(S_{21}^{e e}\right)\right] \\
+\operatorname{Tr}\left[\left(S_{12}^{e e}\right)^{\dagger}\left(S_{11}^{e h}\right)\left(S_{21}^{e h}\right)^{\dagger}\left(S_{22}^{e e}\right)+\left(S_{12}^{e e}\right)^{\dagger}\left(S_{12}^{e h}\right)\left(S_{22}^{e h}\right)^{\dagger}\left(S_{22}^{e e}\right)\right]
\end{aligned}
$$

For example, the third term $\operatorname{Tr}\left[\left(S_{12}^{e e}\right)^{\dagger}\left(S_{11}^{e h}\right)\left(S_{21}^{e h}\right)^{\dagger}\left(S_{22}^{e e}\right)\right]$ corresponds to the two-particle interference of the two processes Fig. 5 (a) and (b). The interference is negative in this case, because it involves the exchange of two electrons (after the Andreev reflection). Again, both processes Fig. 5 (a) and (b) include normal electron reflections, which are forbidden in our case. Other terms also involve similar normal electron reflections. The above analysis shows that the system considered in $[8,34]$ is in a clear distinction from ours, which involves only Andreev reflections (scattering of Cooper pairs).

Let us now turn to the case with $\Gamma_{S}<1$. It turns out that for $\Gamma_{S}<1 / 2$, the cross correlation becomes positive in the limit $2 N / M \Gamma_{S} \gg 1$. This is ascribed to the additional fluctuations at the dot-superconductor interface due to imperfect Andreev reflections, which is forbidden in our system due to the time-reversal symmetry and makes another difference.

It will be useful to simulate this latter effect in our system by applying a magnetic field, which effectively introduces a mass gap into the helical liquid [24].

In conclusion, the close comparisons above reveals where the discrepancy between their and our results arise. While in our setup the cross correlation involves only the scattering of Cooper pairs (or Andreev reflections), in [8] it involves normal electron reflections as well as Andreev reflections. 

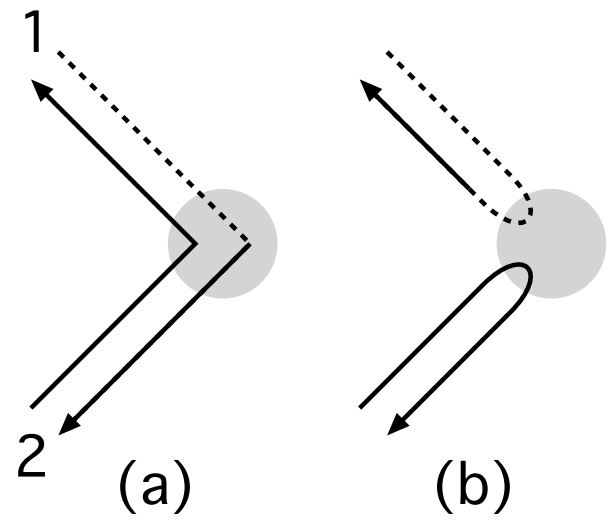

FIG. 5. The third term in Equation (14), $\operatorname{Tr}\left(S_{12}^{e e}\right)^{\dagger}\left(S_{11}^{e h}\right)\left(S_{21}^{e h}\right)^{\dagger}\left(S_{22}^{e e}\right)$, corresponds to the interference of the two processes (a) and (b). The interference is negative since it involves electrons (i.e., the electrons after the AR).

* choims@korea.ac.kr

[1] C. J. Pethick and H. Smith, Bose-Einstein Condensation in Dilute Gases (Cambridge University Press, Cambridge, 2002).

[2] J. Bardeen, L. N. Cooper, and J. R. Schrieffer, Phys. Rev. 108, 1175 (1957).

[3] B. A. Bernevig, T. L. Hughes, and S.-C. Zhang, Science 314, 1757 (2006).

[4] M. Konig, S. Wiedmann, C. Brune, A. Roth, H. Buhmann, L. W. Molenkamp, X.-L. Qi, and S.-C. Zhang, Science 318, 766 (2007).

[5] Strictly speaking, the simple association of bosons with positive and fermions with negative cross correlation is not always true. Nevertheless, the large negative correlation in our results is strongly counter intuitive.

[6] M. P. Anantram and S. Datta, Phys. Rev. B 53, 16390 (1996).

[7] J. Torrès and T. Martin, Eur. Phys. J. B 12, 319 (1999).

[8] P. Samuelsson and M. Büttiker, Phys. Rev. Lett. 89, 046601 (2002).

[9] V. Bouchiat, N. Chtchelkatchev, D. Feinberg, G. B. Lesovik, T. Martin, and J. Torres, Nanotechnology 14, 77 (2003).

[10] L. Hofstetter, S. Csonka, J. Nygard, and C. Schonenberger, Nature 461, 960 (2009).

[11] L. G. Herrmann, F. Portier, P. Roche, A. L. Yeyati,
T. Kontos, and C. Strunk, Phys. Rev. Lett. 104, 026801 (2010).

[12] K. E. Nagaev and M. Büttiker, Phys. Rev. B 63, 081301 (2001).

[13] R. Hanbury Brown and R. Twiss, Nature 177, 27 (1956).

[14] R. Hanbury Brown and R. Twiss, Nature 178, 1046 (1956).

[15] E. Purcell, Nature 178, 1449 (1956).

[16] R. J. Glauber, Phys. Rev. 130, 2529 (1963).

[17] T. Jeltes, J. M. McNamara, W. Hogervorst, W. Vassen, V. Krachmalnicoff, M. Schellekens, A. Perrin, H. Chang, D. Boiron, A. Aspect, and C. I. Westbrook, Nature 445, 402 (2007).

[18] M. Büttiker, Phys. Rev. B 46, 12485 (1992).

[19] M. Henny, S. Oberholzer, C. Strunk, T. Heinzel, K. Ensslin, M. Holland, and C. Schönenberger, Science 284, 296 (1999).

[20] C. L. Kane and E. J. Mele, Phys. Rev. Lett. 95, 146802 (2005).

[21] A. Ström and H. Johannesson, Physical Review Letters 102, 096806 (2009).

[22] C.-Y. Hou, E.-A. Kim, and C. Chamon, Physical Review Letters 102, 076602 (2009).

[23] L. Fu and C. L. Kane, Physical Review Letters 100, 096407 (2008).

[24] L. Fu and C. L. Kane, Phys. Rev. B 79, 161408 (2009).

[25] In the presence of strong (so as for Luttinger liquid parameter to be less than 1/4) electron-electron interaction, the interface barrier can suppress the Andreev reflection. This effect is described elsewhere.

[26] In helical edge modes, the spin- $\uparrow$ (spin- $\downarrow$ ) electron moves only to the right (left). Cooper-pair injection is still poissible: When a Cooper pair is inject, it has a finite centerof-momentum, say, $q$ so that the momenta of two constituting electrons are $q+k$ and $q-k$. One should also recall the $p$-wave nature of the pairing in helical edge modes originating from strong spin-orbit coupling. J. Alicea, Rep. Prog. Phys. 75, 076501 (2012), section 3.

[27] C. Liu, T. L. Hughes, X.-L. Qi, K. Wang, and S.-C. Zhang, Phys. Rev. Lett. 100, 236601 (2008).

[28] K. Sato, D. Loss, and Y. Tserkovnyak, Phys. Rev. Lett. 105, 226401 (2010).

[29] P. Recher and D. Loss, Phys. Rev. Lett. 91, 267003 (2003).

[30] C. W. J. Beenakker, Rev. Mod. Phys. 69, 731 (1997).

[31] Y. M. Blanter and M. Büttiker, Phys. Rep. 336, 1 (2000).

[32] U. Fano, Ame. J. Phys. 29, 539 (1961).

[33] G. Baym, Lectures on Quantum Mechanics (Benjamin, New York, 1974).

[34] P. Samuelsson and M. Büttiker, Phys. Rev. B 66, 201306 (2002). 